\title{
Vortex competition in coastal outflows
}

\author{
Sean Jamshidi ${ }^{1}$ and Edward Johnson ${ }^{1}$ \\ ${ }^{1}$ Department of Mathematics, University College London, \\ London WC1E 6BT, UK. \\ Email: sean.jamshidi.16@ucl.ac.uk (SJ)
}




\begin{abstract}
Experiments and field observations have shown that there are at least two modes of behaviour for river plumes. In many cases, the plume turns to the right (in the Northern hemisphere) on leaving the river mouth and follows the direction of Kelvin-wave propagation. Alternatively, a 'bulge' can form in the plume and a fraction of the outflow volume becomes trapped near the mouth. This paper discusses how bulge formation can be affected by the vorticity profile at the river mouth. Due to the image effect, regions of cyclonic vorticity tend to propagate rightwards, while regions of anticyclonic vorticity propagate leftward upon exit from the source. If an outflow consists of regions of cyclonic vorticity to the left of regions of anticyclonic vorticity, the two image effects are in competition. We explore this phenomenon using a quasi-geostrophic model with piecewise-constant potential vorticity, which allows the vorticity profile at the source to be set as part of the problem. We present analytic solutions valid in the source region and at the head of the plume, and show that all of the outflow travels rightwards if and only if the region of cyclonic vorticity is dominant. The initial-value problem for the model is integrated numerically using the method of contour dynamics, and the full parameter space is explored. We find that if the cyclonic and anticyclonic contributions cancel, as in the experiments of Avicola and Huq (2003), then steady solutions are unstable and a bulge can form downstream of the river mouth.
\end{abstract}

Keywords: Coastal outflows, vorticity dynamics, river plumes. 


\section{Introduction}

Rivers play an important role in the transport of nutrients, sediment and pollutants from the land to the sea, and as such the dynamics of coastal outflow plumes is an active area of study. Outflow plumes that are large enough to be affected by planetary rotation often comprise two distinct parts: a recirculating 'bulge' of fresh water that accumulates near the river mouth, and a coastally-trapped current that transports some or all of the outflow away from the mouth in the direction of Kelvin-wave propagation (Horner-Devine et al., 2015). This prototypical structure has been observed in the Chang Jiang, Hudson and Columbia river plumes (Beardsley et al., 1985; Chant, 2008; Horner-Devine, 2009), as well as in laboratory and numerical studies (Avicola and Huq, 2003; Chen, 2014). However there are also observations of river plumes that do not form a bulge. For example, this is true of the Delaware river (Münchow and Garvine, 1993b;a) and the Chesapeake bay outflow (Donato and Marmorino, 2002). Other plumes may operate in either mode at different times of the year (Conlon, 1982; Shetye et al., 1993).

In cases where a bulge is present, the fraction of the outflow volume that accumulates there is strongly affected by vorticity dynamics. Attempts to predict this fraction based on some measure of vorticity fall into two categories: those that give estimates based on the vorticity of the bulge (Nof and Pichevin, 2001), and those that depend on the vorticity at the source (Fong and Geyer, 2002; Horner-Devine et al., 2006). This work is in the spirit of the second category.

A convenient way to visualise the effect of source vorticity on plume behaviour is through the so-called 'method of images'. A vortex near an impermeable boundary moves as though there is an image vortex, of equal strength and opposite sign, located on the opposite side of the boundary. The image vortex creates a pressure field that advects the physical vortex parallel to the boundary. A cyclonic vortex propagates in the direction of Kelvin-wave flow (rightward in the Northern hemisphere, referred to hereafter as downstream), while an anticyclonic vortex propagates upstream due to this image effect. In the 
context of a coastal outflow, regions of cyclonic vorticity increase transport in the coastal current, while regions of anticyclonic vorticity feed the bulge in the source region, and can even drive fluid upstream in the direction opposite to Kelvin-wave propagation (Johnson et al., 2017). The vorticity profile of a real outflow most likely has regions of positive (cyclonic) vorticity and regions of negative (anticyclonic) vorticity, with the location and strength of these regions changing due to variations in tides, winds and discharge rate.

To illustrate the impact of the spatial variability of vorticity, consider the following simple example: an outflow in the Northern hemisphere consists of a region of positive vorticity on the left, and a region of negative vorticity on the right, looking seaward. Image vorticity drives a tendency for fluid emerging from each region to turn and propagate into the other. Alternatively, if the outflow consists of a region of negative vorticity on the left and a region of positive vorticity on the right, there is no competition and the fluid with negative vorticity turns upstream unimpeded (Garvine, 2001; Magome and Isobe, 2003). In general, we will define a 'competitive region' of an outflow to be any region where positive vorticity lies to the left of negative vorticity, and the behaviour of competitive outflows, i.e., an outflow that is competitive everywhere, is the main subject of this work.

Although it is known that vorticity plays an important role in outflow dynamics, it is not clear how source vorticity can be controlled-or even measured accurately-in a laboratory (see $\S 4.3$ of Crawford (2017) for a discussion of the difficulties involved). In situ measurements of vorticity are even more problematic, and so this work is guided largely by previous laboratory experiments. Avicola and Huq (2003) discuss how differences in experimental set-up can affect source vorticity, suggesting that this is responsible for the discrepancy between their experimental results and those of Klinger (1994). Avicola and Huq (2003) suggest that their outflow has a stronger cyclonic vorticity than in Klinger (1994), and this prevents a bulge from forming as fluid is carried away from the source under a stronger image effect. Instead of measuring vorticity directly, Fong and Geyer (2002) and Horner-Devine et al. (2006) present their results in terms of a source Rossby 
number, the ratio of the source vorticity to the background rotation rate. However, as noted by Horner-Devine et al. (2015), coastal current transport seems to depend only weakly on the source Rossby number and so it is likely that other factors need to be considered. Garvine (2001) shows that plume vorticity can be modified by either topographic stretching or baroclinic effects, but the present model isolates the effect of image vorticity at the source by using a $1 \frac{1}{2}$-layer ocean and a vertical coastline.

\section{a. Idealised models}

One way to avoid the difficulties associated with measuring source vorticity is to use an idealised, inviscid model where the potential vorticity (PV) of the outflow can be specified. Johnson et al. (2017) use a model in which the outflow has the same density as a buoyant upper layer of oceanic fluid. Both the outflow and the upper layer have uniform PV, and the difference between these two values (the PV anomaly, or PVa) governs the dynamics. If the upper layer is deeper than the river mouth (positive PVa) then fluid columns stretch on leaving the source and, by PV conservation, gain vorticity. The source vorticity is cyclonic everywhere and all the outflow travels downstream, leading to a steady, constant-width coastal current and no bulge formation. On the other hand, if the upper layer is shallower than the river mouth (negative PVa) then fluid columns squash on leaving and lose vorticity. In some cases, this leads to an outflow where the source vorticity is negative everywhere and so all fluid turns left, forming an upstream coastal current with only a fraction of the outflow eventually recirculating downstream. Kubokawa (1991) also uses an idealised quasi-geostrophic (QG) model in which the outflow is split between fluids of two different PVs. Part of the outflow has the same PV as the upper ocean layer, and the remainder has a lower PV, so that some regions of the outflow are competitive. Kubokawa shows that possible flows are split into three distinct modes depending on the total volume and the PV distribution of the outflow, these being a steady coastal current, a widening current and an anticyclonic gyre mode. This study builds on these idealised models, generalising the work of Kubokawa (1991) to a wider range of PV distributions, 
and re-analysing the previous results in the framework of vortex competition. In particular, we derive a simple constraint on the source vorticity that must be satisfied in order for the outflow to form a steady plume with no bulge (the coastal current mode).

The model developed here is not appropriate for quantitative comparison with real outflows, due to two important simplifications. Firstly, the QG limit (which is necessary for the analytical progress made below) does not allow for an outcropping density front, so the model ignores density differences between the river plume and the active ambient fluid. However, the vortex dynamics that are study of this work should still play a role in a more realistic setting and may sometimes (for example, in the limit of weak horizontal stratification) be the dominant dynamical factor. It was also shown in Jamshidi and Johnson (2019) that extending the same model to order-one Rossby number has little effect on the qualitative behaviour of the plume. Secondly, we have chosen to model the coast as a vertical wall and are thus ignoring the effect of topographic stretching, which introduces additional cyclonic vorticity into the plume. The role played by topography in plume behaviour is discussed at length in Pimenta et al. (2011), but as it pertains to the present work we note that for a steep bottom slope the plume is known to be 'surface advected' and the influence of topography is small. The relevance of this model in an oceanographic context is discussed further in $\S 6$.

This work is organised as follows. $\S 2$ describes the mathematical model, and determines the range of parameters for a competitive outflow. $\S 3$ presents steady solutions to the long-wave limit of the model, and derives a necessary and sufficient condition for the

existence of the coastal current mode. The full QG equations are solved numerically in $\S 4$, and the unsteady features of these results are discussed in $\S 5$.

\section{Formulation}

Consider flow relative to a frame rotating about a vertical axis $O z$ at constant angular speed $f / 2>0$. Suppose that there is a straight, vertical coast at $y=0$ and consider flow 


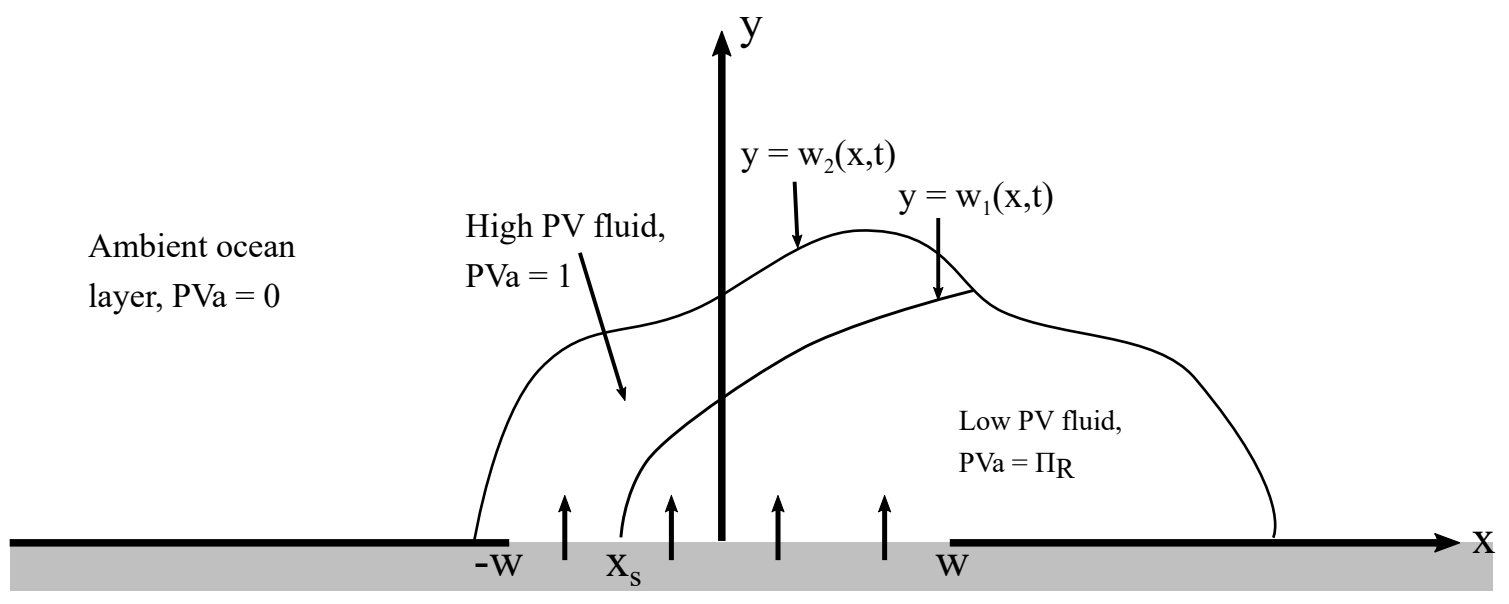

Figure 1: Fluid is expelled from a coastal source located at $|x|<W, y=0$. The ocean fluid in $y \geq 0$ initially consists of an upper, active layer (with the same density as the outflow) on top of a deep, denser, inactive layer. The source vorticity is positive in $x<x_{S}$, and negative in $x>x_{S}$.

in $y \geq 0$ only, with $O x$ directed along the coast. Initially the ocean in $y>0$ is still, with a buoyant upper layer of uniform depth $D$ lying on top of an infinitely deep, dense layer. At time $t=0$ a source in the coast located at $|x|<W$ starts emitting fluid of the same density as the buoyant layer at a rate $Q_{0} D_{S}$ per unit time, where $D_{S}$ is a measure of the source depth and so $Q_{0}$ is the outflow area flux. If $\left|D-D_{S}\right|$ is sufficiently small then the flow is geostrophic everywhere and the motion is governed by the quasi-geostrophic equation for conservation of $\mathrm{PV}$, formulated in terms of $h(x, y, t)$, which measures the departure of the depth interface from the initial value $D$. Under these assumptions, the source vorticity profile can be expressed in terms of the outflow velocity and PVa, which can be chosen in such a way that the outflow is competitive. One simple way to do this is to let the PVa take two values: $\Pi^{*}>0$ in $-W<x<x_{S}^{*}$, and $\Pi_{\mathrm{R}}^{*}<0$ in $x_{S}^{*}<x<W$, where $x_{S}^{*}$ is the location of the dividing streamline in the outflow at which the sign of the vorticity changes. Fluid that exits the outflow to the left of $x_{S}^{*}$ has positive vorticity, and will be referred to as HPVF (high potential-vorticity fluid). The HPVF competes with fluid that exits to the right of $x_{S}^{*}$ and has negative vorticity (LPVF, low potential-vorticity fluid). The choice of piecewise-constant PVa allows for accurate and efficient numerical simulations of the flow using the method of contour dynamics with surgery (Dritschel, 1989), and also facilitates 
the analytical results that follow. A similar model with piecewise constant vorticity was used by Stern and Whitehead (1990) to study the flow of a jet around a corner in a nonrotating environment. A schematic of the situation described above is shown in Figure 1.

We now present the governing equations and boundary conditions for the model, based on the notation and scaling introduced in Johnson et al. (2017) (referred to as JSM hereafter). Horizontal lengths are non-dimensionalised on the source-vortex scale $L_{V}=\left(Q_{0} / \Pi^{*} D_{S}\right)^{1 / 2}$, speeds on $Q_{0} / L_{V}$ and $t$ on the advective time $L_{V}^{2} / Q_{0}=\left(D_{S} \Pi^{*}\right)^{-1}$. The governing equation is therefore

$$
\nabla^{2} \psi-\psi / a^{2}= \begin{cases}0 & \text { in the ambient } \\ 1 & \text { in HPVF } \\ \Pi_{\mathrm{R}} & \text { in LPVF. }\end{cases}
$$

Here, $\psi=g^{\prime} h / f Q_{0}$ is a streamfunction that is related to the non-dimensional velocity by $(u, v)=\left(-\psi_{y}, \psi_{x}\right)$, and $\Pi_{\mathrm{R}}=\Pi_{\mathrm{R}}^{*} / \Pi^{*}$. The parameter $a=L_{R} / L_{V}$ is the non-dimensional Rossby radius, and is discussed in more detail below. The choice of $L_{V}$ for horizontal length-scale is a natural one for the study of vortical effects, as it ensures that the width of the vortically-drive current remains $O(1)$, while the Kelvin-wave decay scale changes with $a$. Later, we will take the long-wave limit of (1), which requires that $L_{V} / W \ll 1$. Note that this does not place any restriction on the Kelvin number, $K=W / L_{R}$, which was introduced by Garvine (1995) as a means of classifying plume behaviour. Outflows with large $K$ have relatively slow flow and are in geostrophic balance, while outflows with small $K$ are little-affected by rotation.

The source is impulsively switched on at $t=0$, with the outflow velocity given by a 
specified profile $Q^{\prime}(x)$. The boundary condition at the coast is therefore

$$
v(x, 0, t)= \begin{cases}0, & |x|>W / L_{V} \\ Q^{\prime}(x), & |x|<W / L_{V}\end{cases}
$$

In the QG limit a Kelvin wave of unit amplitude propagates to the right at infinite speed, setting the coastal interface displacement for all time as

$$
\psi(x, 0, t)=Q(x)
$$

with $Q(x)=1$ for $x>W / L_{V}$ and $Q(x)=0$ for $x<-W / L_{V}$. Although in this work we restrict ourselves to steady mass efflux profiles, the extension to unsteady profiles $Q(x, t)$ follows immediately and is considered by both Kubokawa (1991) and, for the uniform-PV case, in Southwick et al. (2017).

The relative vorticity is given by $\zeta=\nabla^{2} \psi$, so from (1) and (3) the source vorticity profile is completely specified as

$$
\zeta(x, 0, t)=\zeta_{S}(Q)= \begin{cases}1+Q / a^{2} & 0<Q<Q_{+} \\ \Pi_{\mathrm{R}}+Q / a^{2} & Q_{+}<Q<1\end{cases}
$$

where $Q_{+}=Q\left(x_{s}\right)$ is the fraction of the outflow occupied by the HPVF. Note that $\zeta_{S}$ is a function of $Q$, with $x$ appearing parametrically in (4). This means that many of the results below do not depend on the choice of outflow profile $Q(x)$, and is a common feature of 'hydraulic' problems (Pratt and Whitehead, 2008).

Equation (4) shows that the source vorticity in the HPVF is always positive. Some of the LPVF will have negative vorticity if $\Pi_{\mathrm{R}}+Q_{+} / a^{2}<0$, and all of the LPVF has negative vorticity if $\Pi_{R}+1 / a^{2}<0$. Therefore the entire outflow is competitive if and only if $\Pi_{R}<-1 / a^{2}$. Figure 2 illustrates two vorticity profiles: in (a) the whole outflow is 

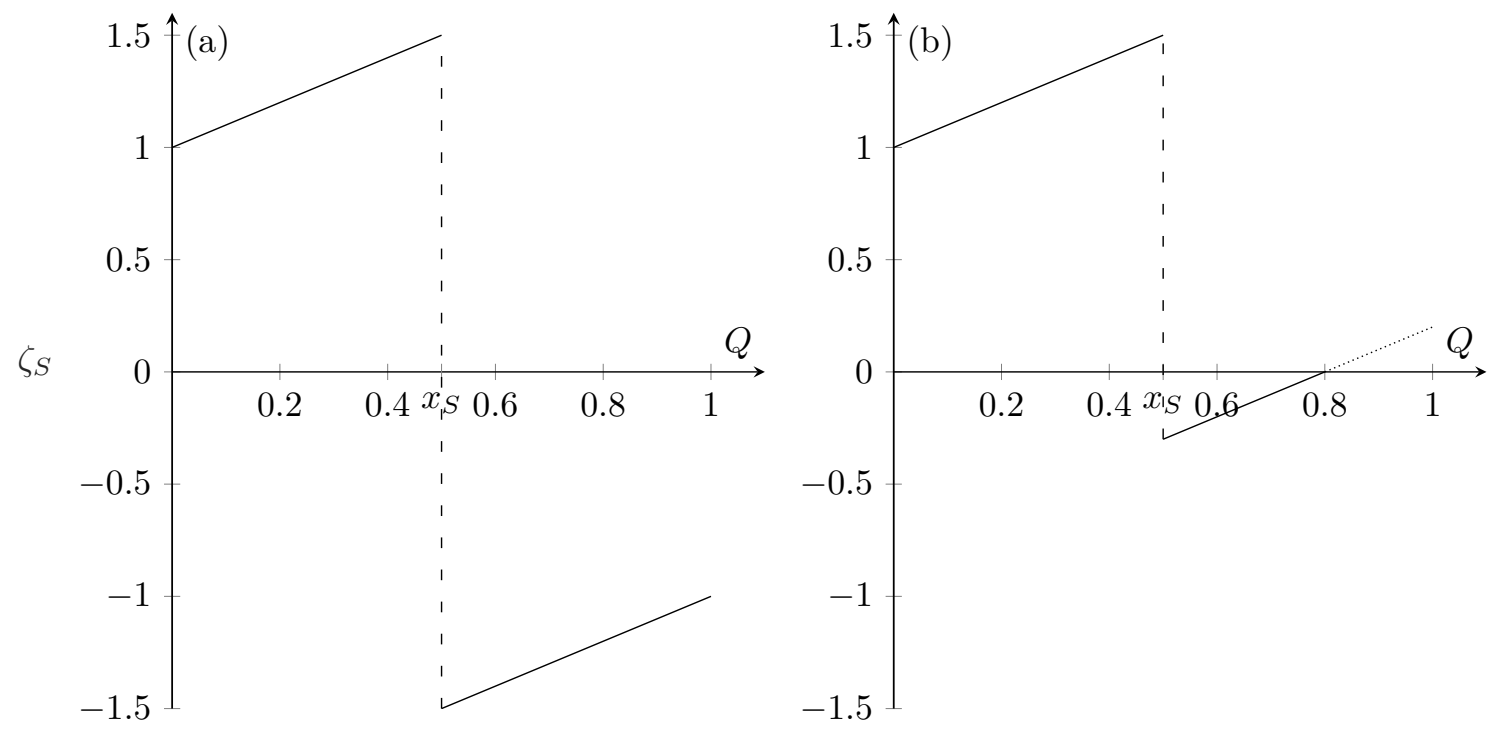

Figure 2: Two examples of the source vorticity profile, $\zeta_{S}(Q)$, with $a=1$ and $Q_{+}=0.5$. (a) $\Pi_{R}=-2>1 / a^{2}$, so that the outflow is competitive. (b) $\Pi_{R}=-0.8<-1 / a^{2}$ so that the outflow is competitive for $Q<0.8$.

competitive, while in (b) the non-competitive region of the LPVF where $\zeta_{S}>0$ is shown dotted.

\section{a. The parameter a}

The present model involves two physical mechanisms. The first is the image effect discussed above, and the second is the flow driven by the Kelvin wave. Although in the QG limit the Kelvin wave itself propagates in the ambient at infinite speed (as reflected in the boundary condition (3)), the flow that it drives in the expelled fluid is of a finite speed, which scales with $1 / a$. If the Rossby number is allowed to be $O(1)$ the Kelvin wave propagates at finite speed. However, once the Kelvin wave has passed a station $x$ the qualitative behaviour at that station is much the same as in the QG limit (Jamshidi and Johnson, 2019). The parameter $a$ therefore measures the relative strengths of Kelvin-wave flow and the image effect, with $a$ larger corresponding to a stronger image effect. The limit $a \rightarrow \infty$ eliminates the Kelvin wave and reduces the governing equations (1) to those of two-dimensional flow (Johnson and McDonald, 2006). On the other hand, $a=0$ is equivalent to an outflow with zero PVa and no image effect to turn the river plume. The 
importance of $a$ is discussed in greater detail in JSM.

\section{The coastal current mode}

JSM show that if the source vorticity is positive everywhere, no bulge forms and all of the outflow travels downstream in a steady, constant-width coastal current. Kubokawa (1991) finds that this 'coastal current mode' occurs if $Q_{+}$is greater than a critical value $Q_{\mathrm{C}}$, which is a function of $\Pi_{\mathrm{R}}$ and $a$. In this section we present solutions that describe the coastal current mode in the long-wave limit of the present model, and show that the critical ratio can be interpreted in terms of a simple condition on the source vorticity.

\section{a. The steady long-wave equations}

The field equation (1) can be solved analytically in the long-wave limit, where disturbances to the interface occur on scales that are much larger in the $x$-direction than in the $y$-direction. Formally, this limit requires that the source is wide compared to the chosen length-scale $L_{V}$, however we are guided by JSM who find that their long-wave theory captures the essential dynamics even in the limiting case where the outflow is modelled as a point source. Therefore let $\epsilon=L_{V} / W$ be small and introduce $X=\epsilon x$ and $T=\epsilon t$, so that the source region is $|X|<1$. Suppose additionally that the boundaries that mark the PV jumps do not overturn, so there are single-valued functions $y=w_{1}(X, T)$ and $y=w_{2}(X, T)$ that denote the boundaries between LPVF and HPVF, and between HPVF and the ambient ocean layer respectively. Under these assumptions, the field equation (1) becomes, at leading order,

$$
\psi_{y y}-\psi / a^{2}= \begin{cases}0 & y>w_{2} \\ 1 & w_{1}<y<w_{2} \\ \Pi_{\mathrm{R}} & 0<y<w_{1} .\end{cases}
$$


Equation (5) is a free-boundary problem to be solved subject to the coastal boundary condition (3) and the far-field condition

$$
\nabla \psi \rightarrow 0 \text { as } y \rightarrow \infty
$$

For ease of notation we will now revert to using the lower-case variables $x$ and $t$. The streamfunction $\psi$ can be written

$$
\begin{aligned}
& \psi_{0}=Q_{e} \mathrm{e}^{-y / a} \quad y>w_{2}, \\
& \psi_{+}=-a^{2}+a^{2} \cosh \left[\left(y-w_{2}\right) / a\right]+Q_{e} \mathrm{e}^{-y / a} \quad w_{1}<y<w_{2}, \\
& \psi_{-}=-a^{2} \Pi_{\mathrm{R}}+A \cosh \left[\left(y-w_{1}\right) / a\right] \\
& +B \sinh \left[\left(y-w_{1}\right) / a\right] \quad 0<y<w_{1},
\end{aligned}
$$

where $A, B$ and $Q_{e}$ are to be determined by continuity conditions at the interfaces and the coastal boundary condition (3). The function $Q_{e}$ is the net flux of ocean fluid at any station $x$.

When the outflow is in coastal current mode, all of the river water travels downstream and so $\psi$ takes the values $Q_{+}$and zero on $y=w_{1}$ and $w_{2}$ respectively. The unique solution in the ambient is therefore $\psi_{0} \equiv 0$ so that $Q_{e}=0$ and the ambient layer is stagnant. The flow in $-1<x<x_{S}$ is unaffected by the region of LPVF, so the solution is exactly the same as for the positive-PVa outflows of JSM. That is, $w_{1}=0$ and

$$
\cosh \left(w_{2} / a\right)=1+Q / a^{2}
$$

Downstream of $x_{S}$, the condition $\psi\left(w_{1}\right)=Q_{+}$is applied to (7):

$$
\begin{aligned}
Q_{+}+a^{2} & =a^{2} \cosh \left[\left(w_{1}-w_{2}\right) / a\right], \\
Q_{+}+a^{2} \Pi_{\mathrm{R}} & =A,
\end{aligned}
$$


so that the width of the HPVF, $w_{2}-w_{1}$, is constant. Note that the negative root of (9) must be taken so that $w_{2}>w_{1}$. The along-shore velocity $u$ is continuous at $y=w_{1}$ so that from (7),

$$
B=a^{2} \sinh \left[\left(w_{1}-w_{2}\right) / a\right]
$$

The coastal boundary condition (3) can then be written in terms of $w_{1}$ alone:

$$
Q+a^{2} \Pi_{\mathrm{R}}=\left(Q_{+}+a^{2} \Pi_{\mathrm{R}}\right) \cosh \left(w_{1} / a\right)+\sqrt{\left(2 a^{2} Q_{+}+Q_{+}^{2}\right)} \sinh \left(w_{1} / a\right) .
$$

Equation (12) only has physically meaningful solutions for certain values of the parameters $a, Q_{+}$and $\Pi_{R}$, and it is these conditions that determine whether the coastal current mode is possible. We will discuss these conditions and their physical meaning presently, but first note that if the conditions are met the solution in the source region is given by

$$
\frac{w_{1}}{a}=\log \frac{F(Q)}{F\left(Q_{+}\right)},
$$

for

$$
F(Q)=a^{2} \Pi_{\mathrm{R}}+Q+\sqrt{Q^{2}+2 a^{2}\left(\Pi_{\mathrm{R}}\left(Q-Q_{+}\right)+Q_{+}\right)} .
$$

This expression is valid in the source region, and gives the offshore location of the internal PV jump as a function of $Q$. The boundary of the outflow plume, $w_{2}$, can be found through (9). Downstream of the source, $Q \equiv 1$ and the coastal current has constant width.

Figure 3 shows two examples of steady long-wave profiles in the source region, plotted as a function of $Q$. In both cases, the dashed curve is $w_{1}$ from (13), and the solid curve is the plume boundary $w_{2}$. Figure $3(\mathrm{~b})$ has $Q_{+}=0.5$ and $\Pi_{\mathrm{R}}=-1-1 / a^{2}$, with these parameter values chosen so that the source vorticity profile is antisymmetric about $x=0$ and the ratio of HPVF to LPVF is $1: 1$. This is the source vorticity profile described in the experiments of Horner-Devine et al. (2006), and consists of a cyclone and anticyclone of equal and opposite strength. Figure 4 shows $w_{A}^{D}$, the width of the coastal current down- 

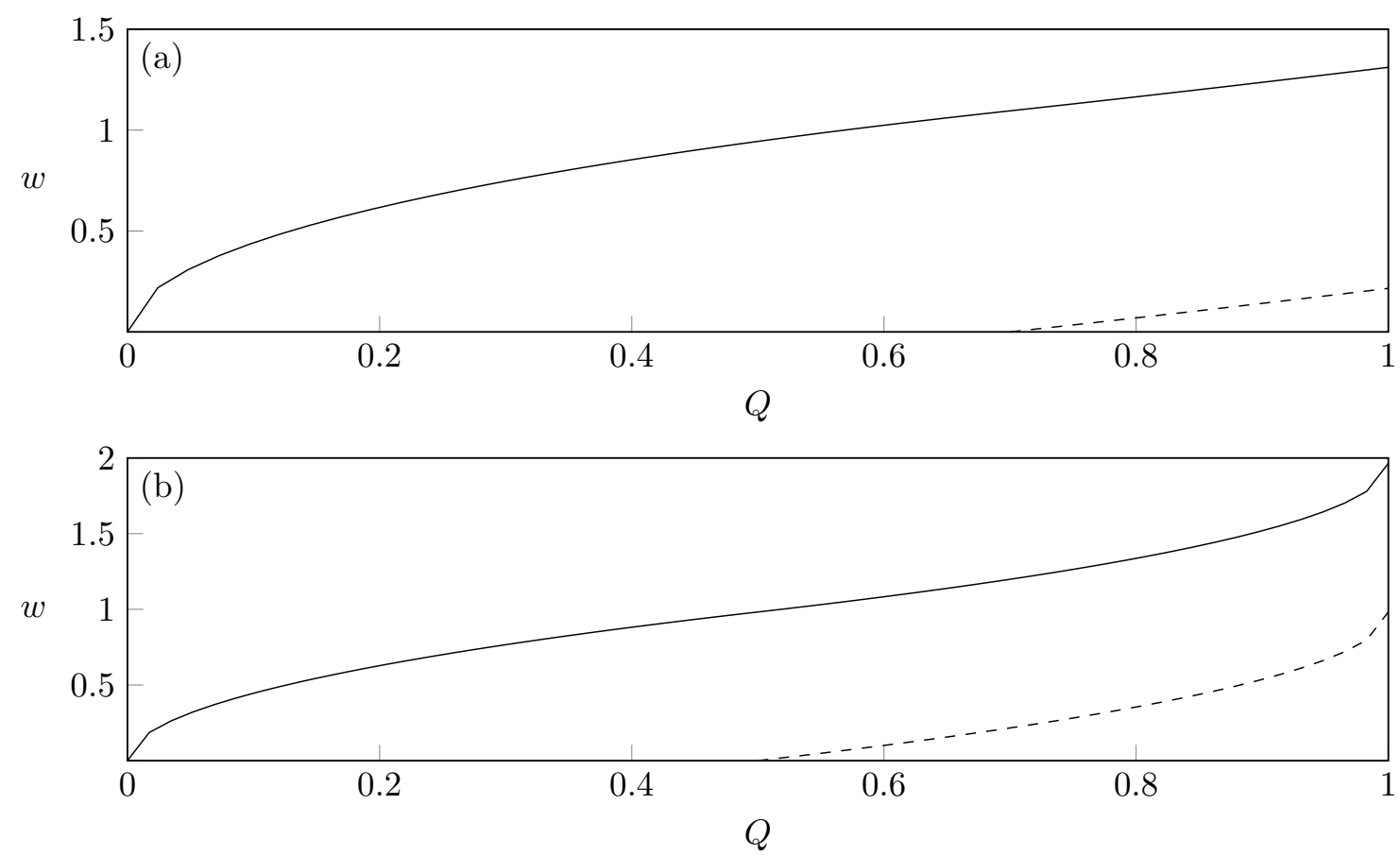

Figure 3: Steady long-wave profiles in the source region. The solid curve shows $w_{2}$, the boundary of the river plume, and the dashed curve is $w_{1}$, the location of the PV jump within the current. (a) $a=0.8, Q_{+}=0.7, \Pi_{\mathrm{R}}=-2$, (b) $a=1.5, Q_{+}=0.5, \Pi_{\mathrm{R}}=-1.44$. 


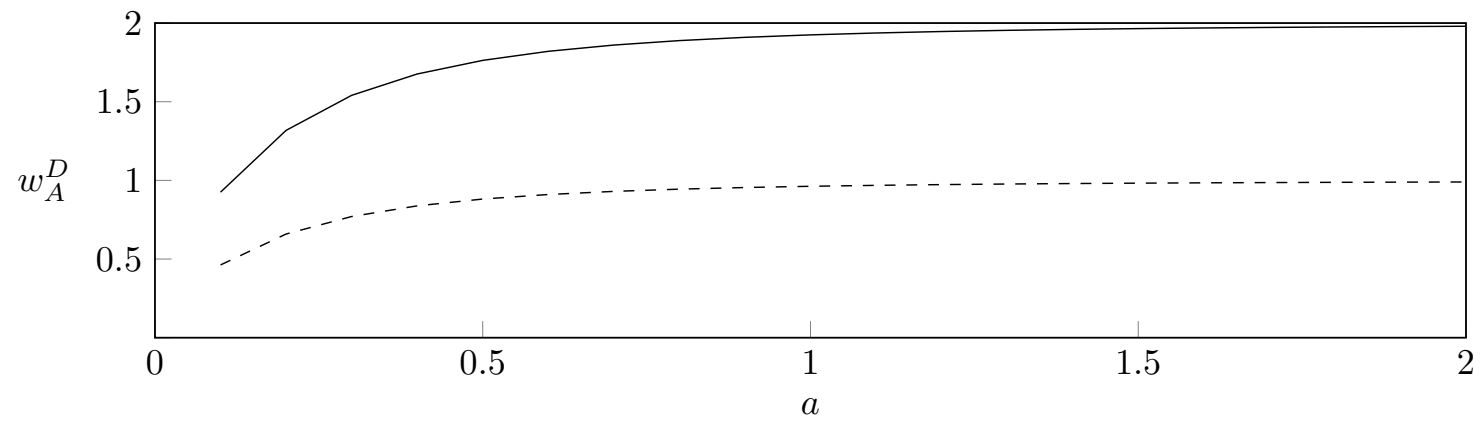

Figure 4: The width of the coastal current, as a function of $a$, for the special case where the source vorticity profile is antisymmetric. The dashed curve shows the width of the interior PV jump.

stream of the source region for the antisymmetric vorticity profile, plotted as a function of $a$. As $a$ increases the image effect becomes stronger relative to the Kelvin-wave driven flow, and the profile approaches the vorticity-dominated limit of Johnson and McDonald (2006), where the current width is equal to twice the vortex-length $L_{V}$.

As noted above, the coastal current mode is only possible under certain conditions. We now derive these conditions by considering the parameter range in which (13) is valid.

i. Competitive outflows For the moment, let us restrict ourselves to the case where the entire outflow is competitive, so that $\Pi_{R}<-1 / a^{2}$. With this condition imposed, (13) is valid provided $F(1)$ is real-valued, or

$$
Q_{+}>Q_{\mathrm{C}}=\frac{\Pi_{\mathrm{R}}+1 / 2 a^{2}}{\Pi_{\mathrm{R}}-1}
$$

Thus, as in Kubokawa (1991) we find that the fraction of the outflow occupied by the HPVF must exceed a critical value $Q_{\mathrm{C}}$ in order for a steady coastal current to form. Outflows dominated by vorticity (either with large $a$ or large $\left|\Pi_{R}\right|$ ) require more HPVF, and a stronger cyclonic vortex, in order to form a coastal current.

The physical meaning of (14) can be seen by re-writing the equation in terms of source vorticity. From (4), $\zeta_{S}$ can be written as a function of $Q$ (equivalently the streamfunction 
$\psi)$ and integrated across the source region:

$$
\int_{0}^{1} \zeta_{S} \mathrm{~d} Q=Q_{+}+\frac{1}{2 a^{2}}+\Pi_{\mathrm{R}}\left(1-Q_{+}\right)
$$

which is positive exactly when $Q_{+}>Q_{\mathrm{C}}$. That is, the coastal current mode occurs if and only if the net contribution of the source vorticity is positive. The first two terms of (15) are positive, and correspond to the downstream flow generated by the cyclonic part of the outflow and the Kelvin wave respectively (since the second term is the only one that depends on the Rossby radius $a$, and vanishes in the vorticity dominated limit of $a \rightarrow \infty$ ). For a steady current to form, the sum of these two must be greater in magnitude than the third term, which is negative and corresponds to the upstream flow generated by the anticyclonic part of the outflow.

ii. Other outflows The integral condition (15) suggests that previous results about coastal current formation in a QG system should be re-analysed with source vorticity in mind. In JSM, the outflow has uniform PVa, $\Pi$. They find that if $\Pi=1$ (and so $\zeta_{S}>0$ everywhere) then the coastal current mode always occurs, while if $\Pi=-1$ the coastal current mode is impossible, although other steady solutions may occur. The second case differs from our set-up since the anticyclonic portion of the outflow is on the left and there is no competition between vortices, resulting in upstream propagation even in steady flow.

In Kubokawa (1991), the outflow is split between fluid with zero PVa on the left of the source and LPVF on the right. If $a>1$ then all of the LPVF has negative vorticity and so the outflow is competitive. In our notation, the vorticity integral becomes,

$$
\int_{0}^{1} \zeta_{S} \mathrm{~d} Q=\int_{0}^{1} \frac{Q}{a^{2}} \mathrm{~d} Q+\int_{Q_{0}}^{1} \mathrm{~d} Q
$$

where $Q_{0}$ is the fraction of the outflow that has zero PVa. Equation (16) is positive if $Q_{0}>1+1 / 2 a^{2}$, which agrees with the condition for coastal current formation given in 
(3.3a) of Kubokawa (1991).

Finally let us now consider the case where only a portion of the outflow is competitive, and the source vorticity is positive at the downstream edge (as in Fig. 2(b)). This situation occurs in the present model if $Q_{+}<\left|a^{2} \Pi_{\mathrm{R}}\right|<1$, in which case the competitive region is $0<Q<\left|a^{2} \Pi_{\mathrm{R}}\right|$. One can show that (13) is valid and the coastal current mode occurs if and only if

$$
\int_{0}^{\left|a^{2} \Pi_{\mathrm{R}}\right|} \zeta_{S} \mathrm{~d} Q>0
$$

so that the critical fraction is $Q_{\mathrm{C}}=a^{2} \Pi_{\mathrm{R}}^{2} / 2\left(1-\Pi_{\mathrm{R}}\right)$.

To summarise the results of this section, we have shown that for a competitive outflow the coastal current mode occurs if and only if the source vorticity profile satisfies an integral condition of the form (15). In fact, there is a simple physical interpretation of this constraint. The element $\zeta \delta Q$ represents the total amount of vorticity contained in a patch of infinitesimal area $\delta Q$ with uniform vorticity $\zeta$. Equation (15) shows that the plume behaves just as the sum of all of these infinitesimal patches, and turns to the right if its 'total vorticity' is positive, as if it were a cyclonic vortex of finite area. Non W-competitive regions of the outflow do not interfere in this process and so are not counted, although of course they still influence the plume strucutre. The general nature of this condition suggests that it might have wider applicability, and an extension to $O(1)$ Rossby number is briefly discussed in the appendix. We also emphasise that the integral condition (15) does not depend on the specific outflow profile, but only on the relative strengths of the cyclonic and anticyclonic parts.

\section{Numerical results}

The full equations (1) (without the long-wave approximation) can be solved using the method of contour dynamics with surgery, which gives fast and accurate results for problems involving piecewise-constant potential vorticity (Dritschel, 1989). In this case, 
the velocity field $\mathbf{u}=(u, v)$ can be written as

$$
\mathbf{u}(\mathbf{x}, t)=-\sum_{k} \Pi_{k} \int_{C_{k}} \mathrm{~K}_{0}\left(\left|\mathbf{x}-\mathbf{x}_{\mathbf{k}}\right| / a\right) \mathrm{d} \mathbf{x}_{\mathbf{k}}
$$

The kernel $\mathrm{K}_{0}$ is the modified Bessel function of the second kind of order zero, which is the appropriate Green's function for the Helmholtz equation (1). The sum is taken over all contours $C_{k}$, where each contour is parameterised by some $\mathbf{x}_{\mathbf{k}}$ and the jump in potential vorticity between contours is given by $\Pi_{k}$. Once the velocity field for the (discretised) contours has been computed numerically, each contour is advected using a standard 4th-order Runge-Kutta scheme. Since the computational speed of this algorithm increases with the square of the number of discretisation points, Dritschel (1989) further employs 'surgery', where small filaments (which do not contribute much to the dynamics) are systematically removed from the main contour. After each advective step, the discrete contours are 'renoded' in such a way that the resolution is proportional to the local curvature. For our particular problem involving a fixed boundary, we must modify (18) to account for contributions from image contours, as well as from the source itself. The contribution from each of these terms is analysed in more detail for a uniform PV outflow in Southwick et al. (2017).

Below, we present results from an initial-value problem where the source is impulsively switched on at $t=0$, and the outflow has a uniform velocity profile $Q^{\prime}(x)=1 / 2$. Simulations must begin with an initial contour, for which we use a thin half-ellipse lying in the source region. Results are insensitive to the choice of initial contour, provided it is sufficiently smooth and covers the entire source.

Figure 5 shows a contour dynamic (CD) run for a competitive outflow in coastal current mode. The parameters are: $a=0.8, Q_{+}=0.7$ and $\Pi_{\mathrm{R}}=-2$, and results are shown at, from top to bottom, $t=15,30,45$. The half-width of the outflow is 3 , so the long-wave parameter $\epsilon=1 / 3$. The plume is shaded, with the LPVF hatched darker. Red dashed curves in the source region show the steady long-wave profiles from $\S 3 \mathrm{a}$, which are in 

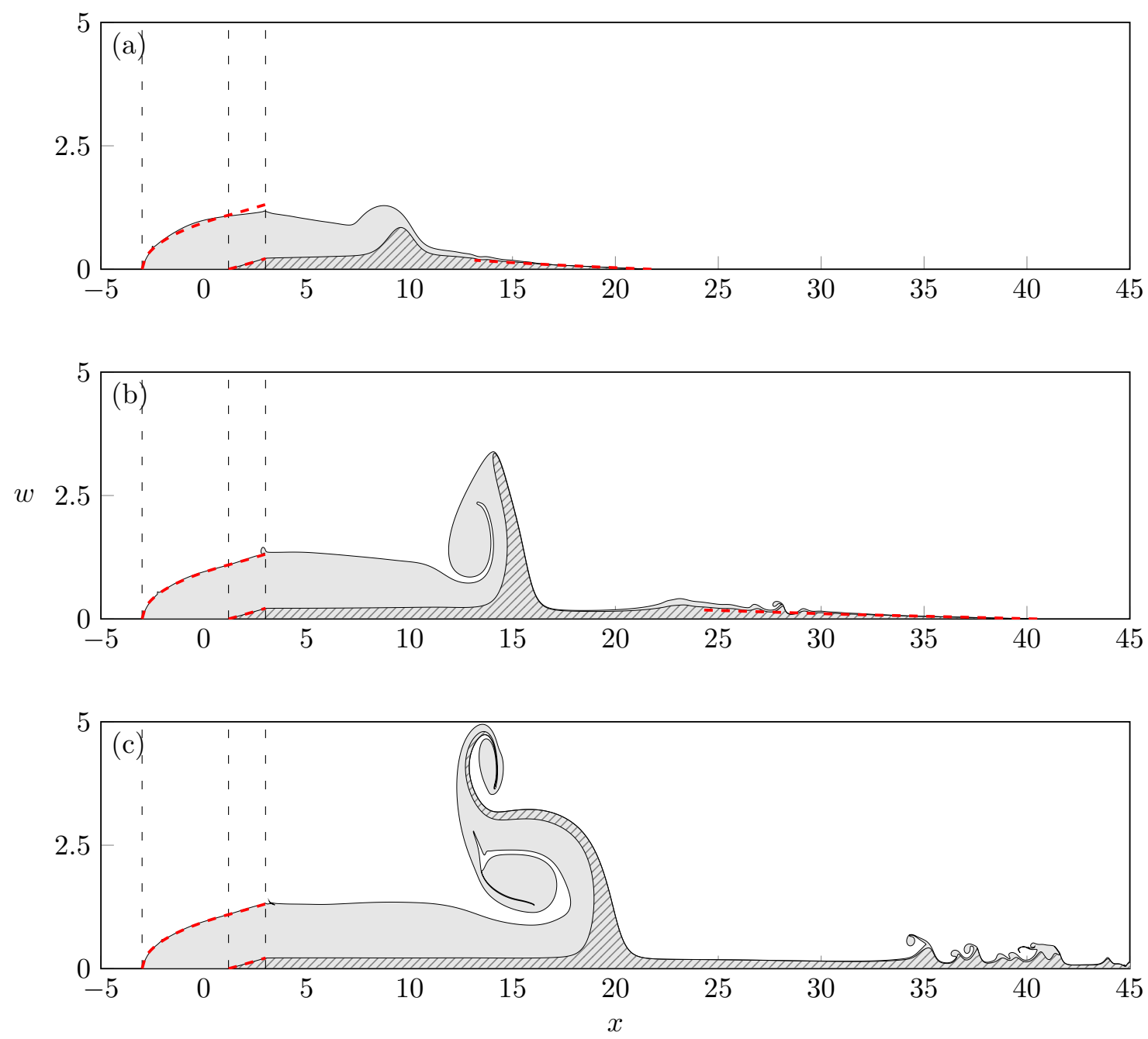

Figure 5: Contour dynamic results for a competitive QG outflow in coastal current mode. The speed ratio $a=0.8$, the fraction of the outflow that has positive vorticity is $Q_{+}=0.7$ and the PV anomaly of the negative vorticity region is $\Pi_{R}=-2$. The half-width of the outflow is 3 , and results are shown at, from top to bottom, $t=15,30,45$. In this and all subsequent figures showing contour dynamics the river plume is shaded, and the LPVF is hatched. Vertical dashed lines mark the source region and $x_{S}$, the point where the source vorticity changes sign. The red dashed lines in the source show the steady long-wave solutions from $\S 3 \mathrm{a}$, and those at the head of the plume in (a) and (b) show the rarefaction computed in $\S 5$ a. 


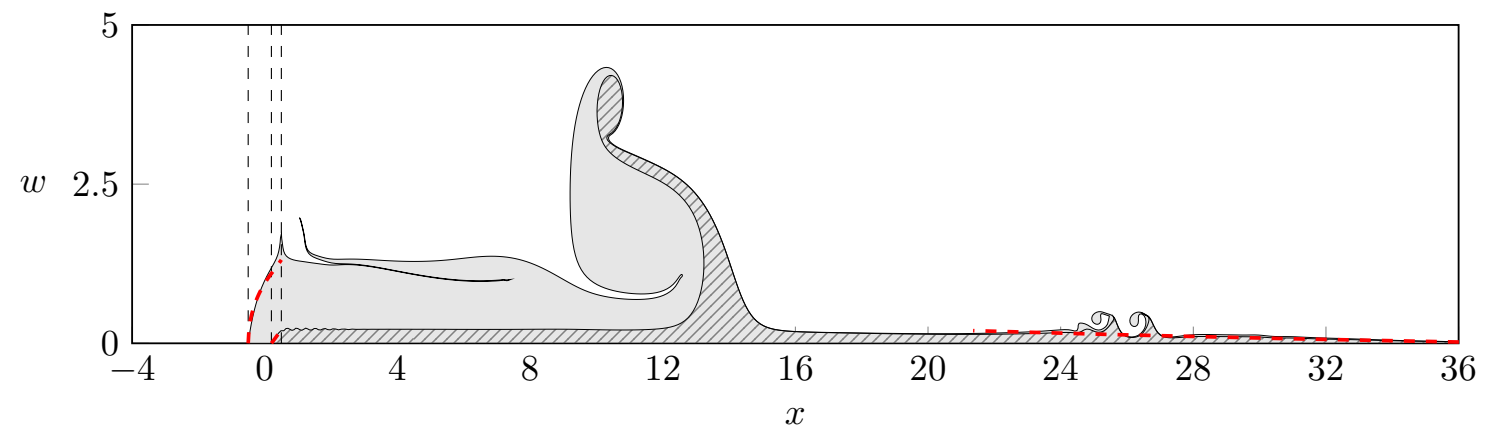

Figure 6: As in Figure 5(b) but for a narrow source with half-width 0.5.

excellent agreement with the numerical results. The coastal current develops quickly: it is almost entirely set-up by $t=15$ and by $t=45$ has extended to $x=15$. At all times the plume is led by a rarefaction consisting only of LPVF, the shape of which is derived below in $\S 5 \mathrm{a}$ and plotted as a red dashed line. Between the rarefaction and the steady current there is an eruption of fluid from the coastal current into the ocean. The eruption initially grows offshore, before later curling up into an eddy that propagates downstream. In (b) and (c), the plume boundary can no longer be written as a single-valued function of $x$ and the analytical results break down. However the cause of the eruption and its initial development can be be qualitatively understood through long-wave theory, and this is done in $\S 5 \mathrm{~b}$. The volume of fluid contained within the eruption increases, and eventually the eruption is strong enough that it retains all of the HPVF (and some of the LPVF) that leaves the source after a certain time. Far away from the site of the eruption the plume is unaffected, so the coastal current and rarefaction are stable features of the plume's evolution.

Figure 6 shows a $\mathrm{CD}$ run that tests the applicability of the long-wave theory to the full QG problem by using a narrow source. The parameters are the same as for Figure 5(b), but with $\epsilon=2$. The overall plume shape appears to be largely unaffected by using a larger value of $\epsilon$, and the analytic results still match the numerics very well.

Figure 7 shows a CD run for an outflow with an antisymmetric vorticity profile. The net contribution from the source vorticity is zero, and the condition (14) is at equality. The steady profile predicted by the long-wave theory is only in partial agreement with 

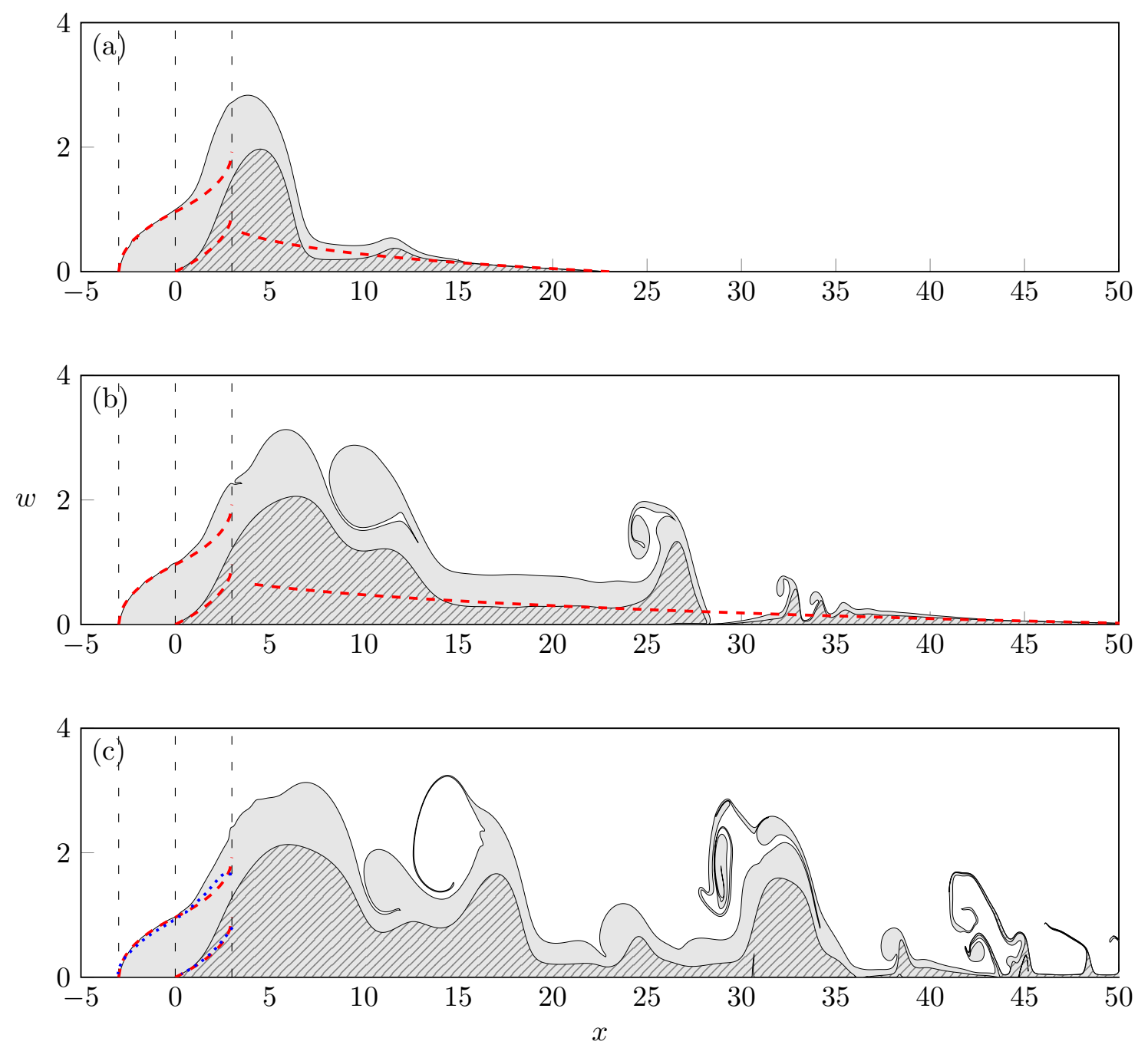

Figure 7: As Figure 5, but for an antisymmetric outflow vorticity profile. The parameters are: $a=1, Q_{+}=0.5, \Pi_{\mathrm{R}}=-2$ and the flow is shown at $t=20,50,60$. Dotted blue curves in (c) show numerically computed steady solutions to the full problem (i.e. without the long-wave approximation).

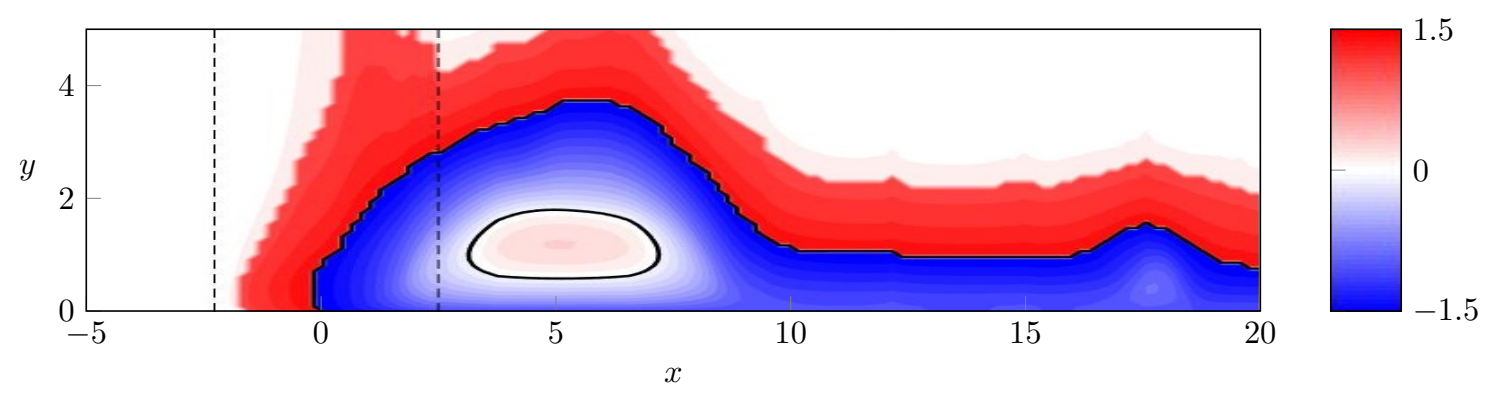

Figure 8: Vorticity contours for the antisymmetric outflow at $t=40$. Black contours show $\zeta=0$. 
the numerics, and no constant-width current occurs. For this set of parameters, the flow immediately downstream of the source is quasi-steady, and there is a bulge of LPVF which appears trapped to the coast. At the head of the plume the rarefaction and the speed of propagation are well-captured by the long-wave theory, but nonlinear, non-periodic waves develop between the head and the bulge at later times. The antisymmetric source vorticity profile is closest to the experiments of Horner-Devine et al. (2006), where the outflow introduces an equal amount of positive and negative vorticity, so it is interesting that this is the only vorticity profile for which we observe a quasi-steady bulge downstream of the source, as is commonly seen in laboratory experiments. Figure 8 plots the vorticity contours at $t=40$. The bulge has strong negative vorticity, and there is a thin layer of positive vorticity around the outside. This structure is in good qualitative agreement with previous experimental results (c.f. Fig. 2 of Horner-Devine et al. (2006)). We note that the steep sections of the plume boundary (for example, at $x \approx 7$ in Fig. 7(a)) are in fact transient features that quickly either erupt into filaments or dissipate, and in general the plume boundary (excluding filaments) does not have steep gradients $\partial w / \partial x$.

To investigate the extent to which the long-wave approximation is responsible for discrepancies between theory and numerics, we ran two further experiments using the same antisymmetric outflow profile. First we conducted another CD run (not shown) with $\epsilon=0.1$. The quasi-steady bulge is still present, although the steady profile agrees with the numerical results over more of the source region. We also used the iterative method described in Southwick et al. (2017) to numerically compute steady solutions to the full problem (i.e. without the long-wave approximation). These are shown as dotted blue curves (Fig. 7(c)) and confirm that steady solutions to the full problem do exist. The stability of these solutions is discussed in the following section.

Figure 9 shows CD results for a competitive outflow that doesn't meet the vorticity integral condition (15), and so by the long-wave theory the coastal current mode is not possible. At early times fluid is directed mainly offshore (Fig. 9(a)), causing the rarefaction 

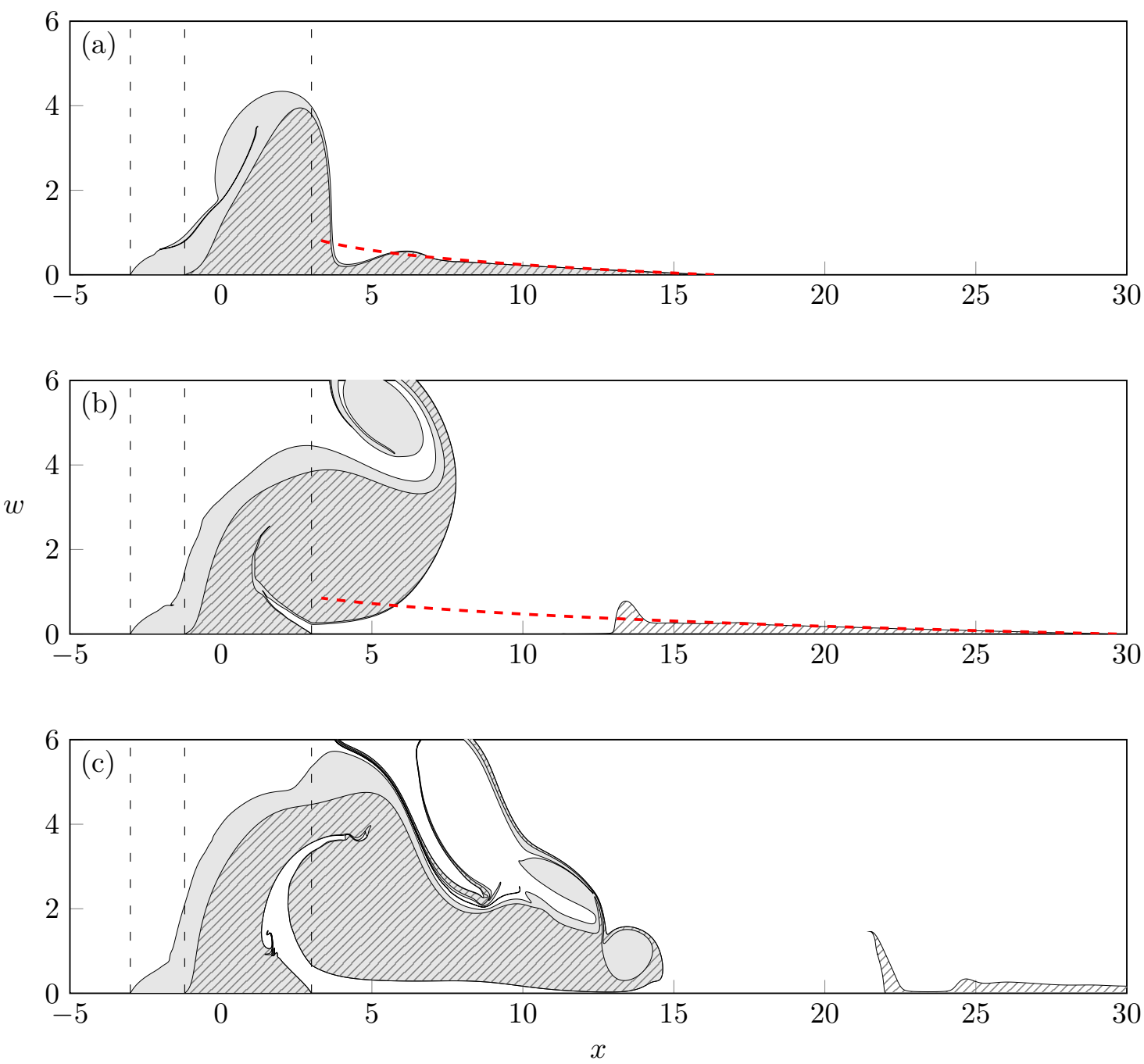

Figure 9: As Figure 5, but for an outflow where the net contribution of the source vorticity is negative. The parameters are: $a=1.5, Q_{+}=0.3, \Pi_{R}=-1$ and the flow is shown at $t=20,40,60$. 
to narrow and eventually pinch off from the bulk. The combined effect of the Kelvin-wave flow and the image of the HPVF is not sufficient to overcome the anticyclonic part of the outflow, and the plume detaches from the coast (as in Stern and Whitehead (1990)). A similar cycle of pinch-off and re-attachment was observed by Horner-Devine et al. (2006) (their Figure 14) in experiments with a low density contrast between the outflow and the ambient.

\section{Unsteady flow features}

In this section, we use properties of the time-dependent long-wave equations to give a qualitative explanation for the eruptions seen in the CD results, as well as a description of the rarefaction and behaviour near the plume nose. The general form of the variable coefficients in the streamfunction (7) is

$$
\begin{aligned}
A & =a^{2}\left(\Pi_{\mathrm{R}}-1\right)+a^{2} \cosh \left[\left(w_{1}-w_{2}\right) / a\right]+Q_{e} \mathrm{e}^{-w_{1} / a}, \\
B & =a^{2} \sinh \left[\left(w_{1}-w_{2}\right) / a\right]-Q_{e} \mathrm{e}^{-w_{1} / a}, \\
Q_{e} & =Q+a^{2} \Pi_{\mathrm{R}}-a^{2}\left(\cosh \left(w_{2} / a\right)+\left(\Pi_{\mathrm{R}}-1\right) \cosh \left(w_{1} / a\right)\right) .
\end{aligned}
$$

The interface locations $w_{1}$ and $w_{2}$ can be found using the kinematic boundary condition

$$
v=\frac{\partial w_{i}}{\partial t}+u \frac{\partial w_{i}}{\partial x} \quad \text { on } y=w_{i}(x, t)
$$

for $i=1,2$. Using the fact that $\psi$ is a streamfunction, this becomes

$$
\frac{\partial w_{i}}{\partial t}=\frac{\partial \psi}{\partial x}+\frac{\partial \psi}{\partial y} \frac{\partial w_{i}}{\partial x}=\frac{\partial}{\partial x} \psi\left(x, w_{i}(x, t)\right)
$$


where

$$
\begin{aligned}
& \psi\left(x, w_{1}\right)=-a^{2}+a^{2} \cosh \left[\left(w_{1}-w_{2}\right) / a\right]+Q_{e} \mathrm{e}^{-w_{1} / a}, \\
& \psi\left(x, w_{2}\right)=Q_{e} .
\end{aligned}
$$

The pair of equations (20) give a first-order, nonlinear hyperbolic system that is forced in the source region by the outflow velocity $Q^{\prime}(x)$. In both the rarefaction and the initial development of the eruption, the system reduces to a single equation with a well-defined wave-speed that allows for a simple interpretation of the CD results.

\section{a. Leading rarefaction}

For all parameter values, there is a region of the plume close to the nose that consists only of LPVF (the 'head' region). Here there is no layer of HPVF between the LPVF and the ambient ocean so we may define $w=w_{2}=w_{1}$. The system (20) reduces to a single equation for $w$ :

$$
\frac{\partial w}{\partial t}+C_{R}(w) \frac{\partial w}{\partial x}=0
$$

where the wave-speed in the head region is

$$
C_{R}(w)=\left(1 / a+a \Pi_{\mathrm{R}}\left(1-\mathrm{e}^{-w / a}\right)\right) \mathrm{e}^{-w / a}
$$

At early stages the downstream plume has $\partial w / \partial x<0$, so a self-similar rarefaction de-

velops if $C_{R}^{\prime}(w)<0$ and small values of $w$ travel faster than larger values. In fact $C_{R}$ is a decreasing function for relevant values of $w$ (i.e. between zero and the width of the coastal current) so that a rarefaction always forms. The nose of the plume moves at speed $C_{R}(0)=1 / a$, which is just the speed of the Kelvin-wave flow. At any time $t$, the rarefaction shape is given by

$$
x-1=C_{R}(w)\left(t-t_{c}(w)\right)
$$


where $t_{c}(w)$ is the time at which the characteristic carrying that value of $w$ emerges from the source region. JSM show that, for the special case of a uniform outflow velocity $Q^{\prime}(x)=1 / 2$, the crossing time $t_{c}=2 a(\exp (w / a)-1)$, and so (25) becomes an implicit

equation for $w$. The rarefaction solution is plotted as a red dashed line in Fig. 5 - 9. There is good agreement in the head region, and the nose always propagates at the predicted speed $1 / a$. Note that the preceding discussion does not require the outflow to be in coastal current mode, and indeed the long-wave theory is accurate near the nose even for the unsteady plume shown in Figure 9.

\section{b. Eruption from the coastal current}

The long-wave theory can also be used to explain eruptions of coastal fluid into the ambient. Although eruptions are seen in all CD runs, they have different characteristics that broadly coincide with whether the net contribution of the source vorticity is positive, zero or negative. For outflows that have net positive source vorticity, the eruption propagates downstream and ejects a thin layer of fluid far into the ocean. In Figure 7, where the source vorticity is antisymmetric, the eruption is bounded and a bulge of LPVF is retained near the source. Finally, if the net contribution from the source vorticity is negative and outflows are not in coastal current mode, then the eruption occurs within the source region. During the early stages of eruption (Fig. 5(a)) $w_{1}$ and $w_{2}$ are displaced by approximately the same amount, so for a first approximation we may consider the characteristic equations (20) with $w_{2}=w_{1}+H$, where the constant $H$ is given by the steady profile (9) as

$$
H=a \cosh ^{-1}\left(1+Q_{+} / a^{2}\right)
$$

With this substitution, the wave-speed is

$$
C_{E}\left(w_{1}\right)=\frac{1}{a}\left(1+a^{2} \Pi_{\mathrm{R}}\left(1-\mathrm{e}^{-w_{1} / a}\right)+\left(\sqrt{Q_{+}\left(2 a^{2}+Q_{+}\right)}-Q_{+}\right) \mathrm{e}^{-w_{1} / a}\right) \mathrm{e}^{-w_{1} / a},
$$


which is similar to (24) apart from an extra term due to interaction between LPVF and HPVF. Since values of $w_{1}$ are conserved along curves moving at speed $\mathrm{d} x / \mathrm{d} t=C_{E}\left(w_{1}\right)$, the initial movement of the eruption is downstream if $C_{E}$ is positive. For antisymmetric outflows where $Q_{+}=1 / 2$ and $\Pi_{\mathrm{R}}=-1-1 / a^{2}$, the wave-speed $C_{E}$ vanishes at the downstream edge of the source causing disturbances to become trapped. A similar situation is discussed in Johnson and Clarke (1999) in the context of topographic control, where they show that a vanishing wave-speed leads to a build up of momentum, and prevents longwave steady profiles from being realised in the CD simulations. This can be understood by considering a small perturbation $\delta(x, t)$ to the steady flow $w_{1}^{S}$. To first order, the kinematic boundary condition (20) becomes

$$
\delta_{t}=\left[\psi\left(w_{1}^{S}\right)-C_{E}\left(w_{1}^{S}\right) \delta\right]_{x},=-\left(C_{E}\left(w_{1}^{S}\right) \delta\right)_{x}
$$

using the fact that in steady flow $\psi\left(w_{1}^{S}\right)=Q_{+}$is constant. Multiplying (28) by $C_{E}$ shows that the perturbation momentum $C_{E} \delta$ is conserved and so as $C_{E} \rightarrow 0$ the amplitude $\delta$ becomes arbitrarily large. Therefore, in an outflow where the net contribution of the source vorticity is zero, the steady long-wave profiles of $\S 3$ a are unstable to small perturbations and so will never be seen in the initial value problem.

Figure 10(a) shows two representative examples of $C_{E}\left(w_{1}\right)$ corresponding to the $\mathrm{CD}$ runs presented in $\S 4$, with the coastal current mode of Figure 5 shown as a solid curve and the antisymmetric outflow of Figure 7 as a dashed curve. The maximum width of $w_{1}^{S}$ is marked with a circle. Both curves have a negative gradient, so any perturbation to steady flow will steepen on the upstream side as smaller values of $w_{1}$ move faster. However note that for the antisymmetric profile the curve flattens as $w_{1}$ increases, so $\partial w_{1} / \partial x$ remains $O(1)$ and the eruption in Figure 7 is bounded. On the other hand the solid curve corresponding to Figure 5 is positive at the downstream edge and has a steeper gradient, so the eruption propagates downstream and is relatively strong, as is discussed in Stern (1986). To further examine bulge formation in antisymmetric outflows, Figure 10(b) 

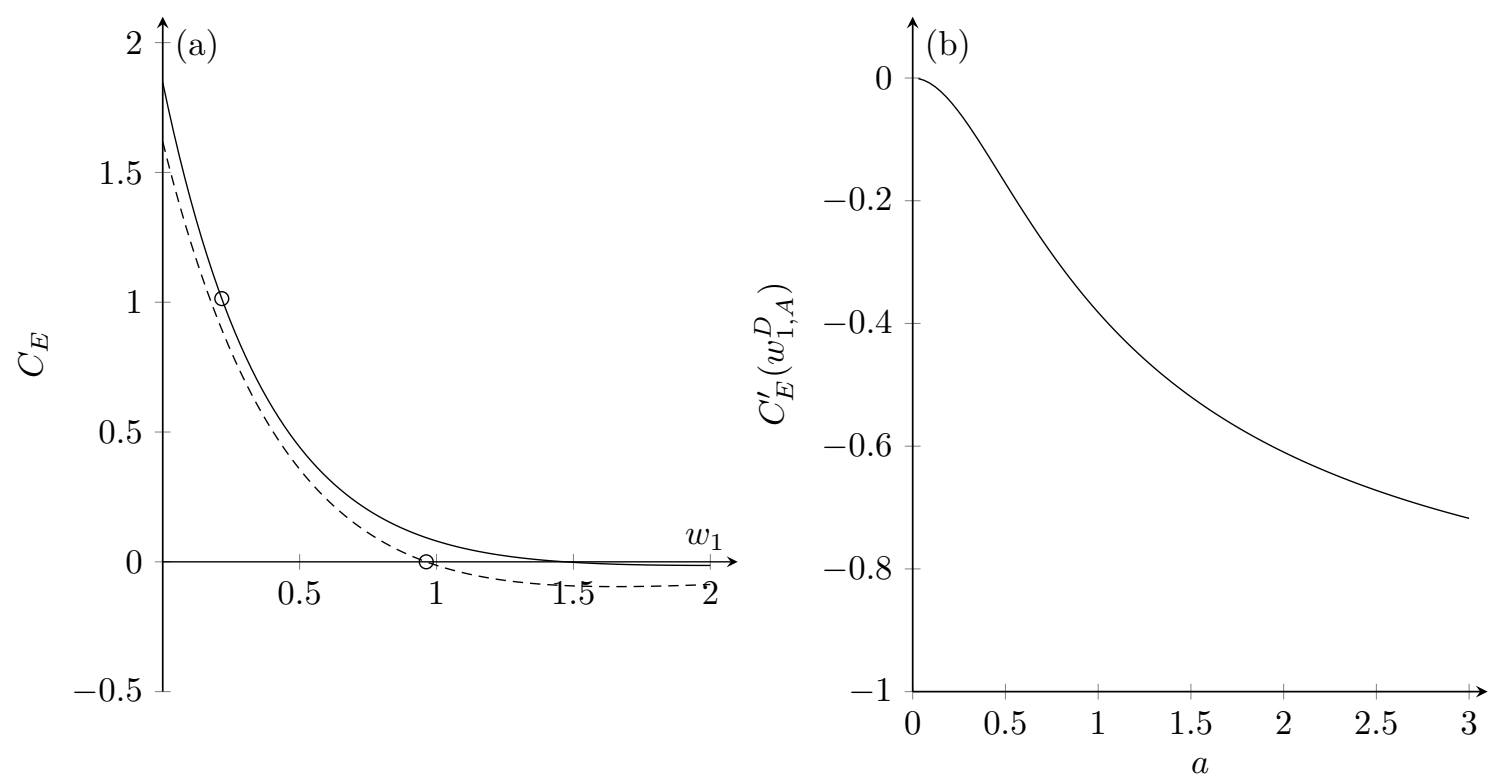

Figure 10: (a) The long-wave speed (27) at the early stages of an eruption. The solid line uses the same parameters as the coastal current mode of Figure 5; and the dashed line uses the same as the antisymmetric profile of Figure 7. Circles mark the downstream width of the steady profile. (b) The slope of $C_{E}$ at the downstream width $w_{1, A}^{D}$ for antisymmetric profiles, as a function of $a$.

plots the slope of $C_{E}$ at the downstream edge of the source. As $a$ increases and vorticity becomes more dominant, $\left|C_{E}^{\prime}\left(w_{1, A}\right)\right|$ is larger, so waves steepen more and the eruption is no longer bounded. Thus, in the QG model, it seems that quasi-steady bulges can only exist in antisymmetric outflows where vortical effects are relatively weak.

We briefly consider the behaviour of the outflow in Figure 9, where the net contribution from the source vorticity is negative and the coastal current mode is not possible. Instead, the profile given by equation (13) is only valid over part of the source region, with the radical vanishing and $F(Q)$ becoming complex at $Q=0.73$. Differentiating (13) gives

$$
\frac{\partial w_{1}}{\partial x}=\frac{a Q^{\prime}(x)}{\sqrt{ }\left(Q^{2}+2 a^{2}\left(\Pi_{\mathrm{R}}\left(Q-Q_{+}\right)+Q_{+}\right)\right)},
$$

so that the loss of the steady solution is associated with an infinite gradient in the profile and, as in Stern (1986), a strong eruption of coastal fluid that here detaches completely from the coast. 


\section{Summary and conclusions}

Numerical and theoretical results for coastal outflows have been discussed in terms of a quasi-geostrophic model which allows for complete control over the source vorticity profile by specifying the PV distribution of the expelled fluid. This is the key feature of this work, as it gives insight into the relationship between the vorticity of the outflow and the resulting plume structure. In particular, we are interested in the case of a 'competitive outflow', where negative vorticity is on the right of the source and positive vorticity is on the left, as in this situation the image effect imparts a tendency for cyclones and anticyclones to pass through each other. Analytical steady solutions to the long-wave equations have been derived, and it is shown in $\S 3$ that the coastal current mode is only possible if the net contribution of the source vorticity (the integral of $\zeta$ with respect to the streamfunction $\psi$ over competitive regions of the outflow) is positive. It is encouraging that the results from Kubokawa (1991) agree with our, more general, condition on the outflow. Kubokawa's results do not depend on the long-wave approximation and so this suggests that the source vorticity condition is applicable even when $\epsilon=O(1)$.

The numerical results of $\S 4$ confirm that when the region of cyclonic vorticity is dominant all fluid turns to the right after leaving the source (Fig. 5) and a stable coastal current develops. The plume nose consists entirely of LPVF and propagates at the speed of the Kelvin-wave driven flow, $Q_{0} / L_{R}$ (where $Q_{0}$ is the area flux of the outflow). The head of the intrusion is a self-similar rarefaction, which can be described by long-wave theory. If the net contribution of source vorticity is negative, the anticyclonic region is dominant and the coastal current mode is not possible. The plume cannot remain attached, and separates from the coast as it leaves the source (Fig. 9). The transition case, where the cyclonic and anticyclonic contributions cancel, is closest to the laboratory experiments of Avicola and Huq (2003) and Horner-Devine et al. (2006). A steady long-wave profile exists but it is unstable to small perturbations and, depending on the value of $a$, the plume can develop a quasi-steady bulge just downstream of the source (Fig. 7). The bulge forms due to the 
accumulation of short-wave energy, and appears to reduce the downstream transport of LPVF. It is notable that these features are only seen in runs where the net vorticity is zero.

\section{a. Oceanographic context}

It is suggested in Avicola and Huq (2003) and by Chen (2014) that fluid from the right-hand side of the outflow tends to gather in the bulge, so that both the sign and the distribution of source vorticity play a role in the initial development of the plume, and therefore contribute to the overall structure. However in situ measurements of plume vorticity are rare, particularly in the source region, although there is much evidence that where bulges exist they are anticyclonic (for example in Figure 5 of Chant (2008)). This work has therefore been guided by laboratory plumes and, despite the simplicity of the model, has reproduced many of their qualitative features, with a bulge core that consists of negative vorticity, and a thin layer of positive vorticity at the plume boundary and in the coastal current. However, modelling the outflow as a rectangular channel (as is done here and in many experiments) ignores variations in topography and estuary shape, which surely affect the behaviour of the plume. This is discussed at length in the outflow context by Pimenta et al. (2011), and for flow around a cape by Lin et al. (2018), who show that separation of the East Greenland coastal current is caused by widening isobaths. We should also emphasise that the principal result (15) applies only to outflows with a competitive vorticity structure, which may not always be the case. In fact, the source velocity profile of the Delaware river from Münchow and Garvine (1993a) features landward flow on the left-hand side of the estuary, so that the vorticity is cyclonic throughout.

We hope that the discussion of vortical effects presented here is also relevant for the more general problem of flow through a sea strait or around a cape. Whitehead and Miller (1979) and Bormans and Garrett (1989) discuss flow through the Strait of Gibraltar, where relatively fresher Atlantic water flows eastward and forms an anticyclonic gyre in the Alboran sea. Bormans and Garrett (1989) suggest that the flow turns through an inertial circle, and so separates from the coast to form a gyre if the inertial radius $U / f$ is greater than the 
radius of curvature at the corner of the strait. In this inertial framework, where vortex effects are absent, a gyre always forms when the flow is around a sharp corner. The QG model used here balances the Coriolis term with pressure gradient, so vorticity dynamics dominate over inertia and gyre formation is relatively rare. A more nuanced treatment of rotational flow around a corner, that includes contributions from relative vorticity, inertia and topography is an important avenue for further work.

\section{APPENDIX}

Jamshidi and Johnson (2019) discuss the outflow problem for $O(1)$ Rossby number using a semi-geostrophic (long-wave) model with uniform PV. Here we consider the equivalent set-up for a competitive outflow, and show that the coastal current mode is only possible if the net contribution from the source vorticity is positive.

The governing equations for semi-geostrophic flow are:

$$
\begin{aligned}
\frac{\partial u}{\partial t}+u \frac{\partial u}{\partial x}+v \frac{\partial u}{\partial y}-v & =-\frac{\partial h}{\partial x} \\
u & =-\frac{\partial h}{\partial y} \\
\frac{\partial h}{\partial t}+\frac{\partial}{\partial x}(u h)+\frac{\partial}{\partial y}(v h) & =0
\end{aligned}
$$

where, following Jamshidi and Johnson (2019), $x$ has been non-dimensionalised with $W$, $y$ with $L_{R}, u$ and $v$ with $\sqrt{g^{\prime} D_{S}}$ and $\sqrt{g^{\prime} D_{S}}\left(L_{R} / W\right)$ respectively, $h$ with $D_{S}$ and $t$ with $W / \sqrt{g^{\prime} D_{S}}$. The long-wave parameter is $L_{R} / W$ and is assumed to be small (note that this is different to $\epsilon=L_{V} / W$ in the QG model). The potential vorticity $q$ is piecewise constant, and takes non-dimensional values $1 / H, 1$, and $q_{\mathrm{R}}$ in the ambient ocean, the HPVF and the LPVF respectively. The presence of an ambient ocean-layer with $H>1$ is a necessary requirement in this model for the plume to have $\zeta>0$ at $y=w_{2}$. From conservation of 
PV and continuity of $h$ and $u$, the layer-depth $h$ is

$$
h= \begin{cases}1+(H-1) \cosh \left(y-w_{2}\right) & w_{1}<y<w_{2} \\ \frac{1}{q_{\mathrm{R}}}+A \cosh \left[\sqrt{q_{\mathrm{R}}}\left(y-w_{1}\right)\right]+B \sinh \left[\sqrt{q_{\mathrm{R}}}\left(y-w_{1}\right)\right] & y<w_{1},\end{cases}
$$

where

$$
\begin{aligned}
& A=\frac{q_{\mathrm{R}}-1}{q_{\mathrm{R}}}+(H-1) \cosh \left(w_{1}-w_{2}\right), \\
& B=(H-1) \sinh \left(w_{1}-w_{2}\right) / \sqrt{q_{\mathrm{R}}} .
\end{aligned}
$$

As in the QG model, the ambient ocean is undisturbed. The interface widths $w_{1}$ and $w_{2}$ are determined by the flux conditions

$$
\begin{aligned}
Q_{0} & =\int_{0}^{w_{2}} u h \mathrm{~d} y=\frac{h_{S}^{2}-H^{2}}{2}, \\
Q_{+} & =\int_{w_{1}}^{w_{2}} u h \mathrm{~d} y=\frac{h_{1}^{2}-H^{2}}{2},
\end{aligned}
$$

where $Q_{0}$ is the non-dimensional volume flux of the outflow, and $h_{S}$ and $h_{1}$ are the layerdepths at the coast and $w_{1}$ respectively. The expressions for $w_{1}$ and $w_{2}$ are complicated and not particularly instructive, but can be found computationally using symbolic manipulation. For a given $Q_{0}$ and $H$ we test values of $Q_{+}$and $q_{\mathrm{R}}$ to numerically determine the region of the parameter space where $w_{1}$ is valid and so the coastal current mode is possible. For the particular case of $Q_{0}=1.5, H=2$, the region of the parameter space where a coastal current is possible is shown shaded in Figure 11. 


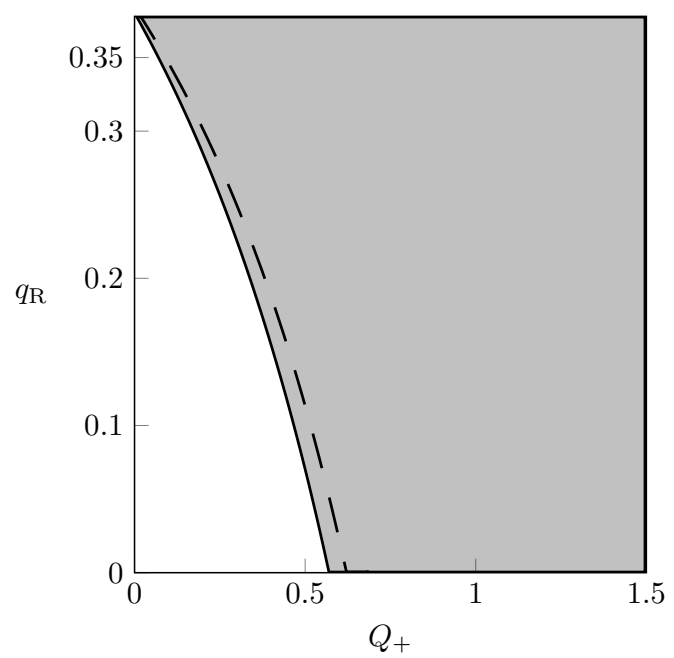

Figure 11: Numerically computed region of the $\left(Q_{+}, q_{\mathrm{R}}\right)$ parameter space for which the coastal current mode exists (shaded grey). The integrated source vorticity is positive to the right of the dashed line. The outflow parameters are $Q_{0}=1.5$ and $H=2$.

We then compute the source vorticity integral analytically.

$$
\begin{aligned}
\int_{\text {source }} \zeta_{S} \mathrm{~d} Q & =\int_{0}^{Q_{0}} q h_{S}-1 \mathrm{~d} Q \\
& =-Q_{0}+\int_{0}^{Q_{+}} h_{S} \mathrm{~d} Q+q_{\mathrm{R}} \int_{Q_{+}}^{Q_{0}} h_{S} \mathrm{~d} Q \\
& =-Q_{0}-\frac{H^{3}}{3}+\frac{1-q_{\mathrm{R}}}{3}\left(H^{2}+2 Q_{+}\right)^{3 / 2}+\frac{q_{\mathrm{R}}}{3}\left(H^{2}+2 Q_{0}\right)^{3 / 2}
\end{aligned}
$$

where the last step has made use of (32). The region to the right of the dashed line in Figure 11 shows where the net contribution is positive. There is good agreement between these two regions, so again it seems that the coastal current mode can occur if and only if the net contribution of source vorticity is positive.

\section{REFERENCES}

Avicola, G. and P. Huq (2003), 'The role of outflow geometry in the formation of the recirculating bulge region in coastal buoyant outflows', J. Mar. Res. 61(4), 411-434. 
Beardsley, R. C., R. Limeburner, H. Yu and G. A. Cannon (1985), 'Discharge of the Chang Jiang (Yangtze river) into the East China sea', Cont. Shelf Res. 4(1-2), 57-76.

Bormans, M. and C. Garrett (1989), 'A simple criterion for gyre formation by the surface outflow from a strait, with application to the Alboran sea', J. Geophys. Res. - Oceans 94(C9), 12637-12644.

Chant, R. J. et al (2008), 'Bulge formation of a buoyant river outflow', J. Geophys. Res. Oceans 113(C1).

Chen, S. (2014), 'Enhancement of alongshore freshwater transport in surface-advected river plumes by tides', J. Phys. Oceanogr. 44(11), 2951-2971.

Conlon, D. M. (1982), 'On the outflow modes of the tsugaru warm current', La mer 20, 6064.

Crawford, T. J. (2017), An experimental study of the spread of buoyant water into a rotating environment, $\mathrm{PhD}$ thesis, University of Cambridge.

Donato, T. F. and G. O. Marmorino (2002), 'The surface morphology of a coastal gravity current', Cont. Shelf Res. 22(1), 141-146.

Dritschel, D. G. (1989), 'Contour dynamics and contour surgery: numerical algorithms for extended, high-resolution modelling of vortex dynamics in two-dimensional, inviscid, incompressible flows', Comput. Phys. Rep. 10(3), 77-146.

Fong, D. A. and W. R. Geyer (2002), 'The alongshore transport of freshwater in a surfacetrapped river plume', J. Phys. Oceanogr. 32(3), 957-972.

Garvine, R. W. (1995), 'A dynamical system for classifying buoyant coastal discharges', Cont. Shelf Res. 15(13), 1585-1596.

Garvine, R. W. (2001), 'The impact of model configuration in studies of buoyant coastal discharge', J. Mar. Res. 59(2), 193-225. 
Horner-Devine, A. R. (2009), 'The bulge circulation in the Columbia River plume', Cont. Shelf Res. 29(1), 234-251.

Horner-Devine, A. R., D. A. Fong, S. G. Monismith and T. Maxworthy (2006), 'Laboratory experiments simulating a coastal river inflow', J. Fluid Mech. 555, 203-232.

Horner-Devine, A. R., R. D. Hetland and D. G. MacDonald (2015), 'Mixing and transport in coastal river plumes', Annu. Rev. Fluid Mech. 47, 569-594.

Jamshidi, S. and E. R. Johnson (2019), 'Coastal outflow currents into a buoyant layer of arbitrary depth', J. Fluid Mech. 858, 656-688.

Johnson, E. R. and N. R. McDonald (2006), 'Vortical source-sink flow against a wall: The initial value problem and exact steady states', Phys. Fluids 18(7), 076601.

Johnson, E. R. and S. R. Clarke (1999), 'Dispersive effects in Rossby-wave hydraulics', J. Fluid Mech. 401, 27-54.

Johnson, E.R., O.R. Southwick and N.R. McDonald (2017), 'The long-wave vorticity dynamics of rotating buoyant outflows', J. Fluid Mech. 822, 418-443.

Klinger, B. A. (1994), 'Baroclinic eddy generation at a sharp corner in a rotating system', J. Geophys. Res. - Oceans 99(C6), 12515-12531.

Kubokawa, A. (1991), 'On the behaviour of outflows with low potential vorticity from a sea strait', Tellus A 43(2), 168-176.

Lin, P., R. S. Pickart, D. J. Torres and A. Pacini (2018), 'Evolution of the freshwater coastal current at the southern tip of Greenland', J. Phys. Oceanogr. 48(9), 2127-2140.

Magome, S. and A. Isobe (2003), 'Current structure and behavior of the river plume in suo-nada', J. Oceanogr. 59(6), 833-843. 
Münchow, A. and R. W. Garvine (1993a), 'Dynamical properties of a buoyancy-driven coastal current', J. Geophys. Res. - Oceans 98(C11), 20063-20077.

Münchow, A. and R.W. Garvine (1993b), 'Buoyancy and wind forcing of a coastal current', J. Mar. Res. 51(2), 293-322.

Nof, D. and T. Pichevin (2001), 'The ballooning of outflows', J. Phys. Oceanogr. 31(10), 3045-3058.

Pimenta, F. M., A. D. Kirwan Jr and P. Huq (2011), 'On the transport of buoyant coastal plumes', J. Phys. Oceanogr. 41(3), 620-640.

Pratt, L. J. and J. A. Whitehead (2008), Rotating Hydraulics, Vol. 1, Springer.

Shetye, S. R., A. D. Gouveia, S. S. C. Shenoi, D. Sundar, G. S. Michael and G. Nampoothiri (1993), 'The western boundary current of the seasonal subtropical gyre in the Bay of Bengal', J. Geophys. Res. - Oceans 98(C1), 945-954.

Southwick, O. R., E. R. Johnson and N. R. McDonald (2017), 'Potential vorticity dynamics of coastal outflows', J. Phys. Oceanogr. 47(5), 1021-1041.

Stern, M. E. (1986), 'On the amplification of convergences in coastal currents and the formation of squirts', J. Mar. Res. 44(3), 403-421.

Stern, M. E. and J. A. Whitehead (1990), 'Separation of a boundary jet in a rotating fluid', J. Fluid Mech. 217, 4169.

Whitehead, J. A. and A. R. Miller (1979), 'Laboratory simulation of the gyre in the Alboran sea', J. Geophys. Res. - Oceans 84(C7), 3733-3742. 